\title{
GREENWAY IN ITALY: EXAMPLES OF PROJECTS AND IMPLEMENTATION
}

\author{
Pasquale Dal Sasso, Maria Antonella Ottolino
}

\section{Introduction}

The huge territorial resources available, featuring environmental and landscape traits, and the sensitivity towards their preservation and conservation, led to the origin and development of the "greenways" concept in the United States [Ahern 1995; Fabos 1995; Turner 2001].

Evolutions and research activities on the meaning and definition of greenways have allowed solutions to be experimented with regards to the interrelation and arrangement of "networked" systems of "green spaces" and infrastructural systems or natural linear elements, to tackle issues encountered in the planning of "wide" areas and "open territories", also involving territories belonging to more than one State [Fabos 2004; Fabos and Ryan 2004].

Expanding the idea of greenways to include land planning also meets the current need to combine social and economic requirements with the amount of human and natural resources available and present in the territory, in a way to draw the most benefits without neglecting the possibility of valorisation and growth of the special features and characteristics of the same territories.

The application of the concept of greenway to the territory and its interaction with the systems of "green spaces" also allows us to tackle issues relating to the control of urban growth, thus suggesting solutions that balance social needs with the needs pertaining to urban and extra-urban territories [Taylor 1995; Yokohari 2006].

In Europe, greenways tend to make use of pre-existing infrastructural systems serving as routes for slow traffic or as an educational tool to learn about

Paper received 27.05.2010; accepted 17.11.2010

Prof. Ing. Pasquale Dal Sasso, PhD Ottolino Maria AntonelLA: Department of Engineering and Management of the Agricultural Systems, Livestock and Forest (PROGESA), University of Bari, via Amendola 165/A 70126 Bari (Italy). Email: dalsasso@agr.uniba.it, tel. 0039.0805442962 fax 0039.0805442955

The paper is to be attributed to the authors in equal measure. and protect the cultural, historical and landscape heritage [European Greenways Association 1999].

A constant and unchanged characteristic in the definitions of greenway lies in any case in the presence of pre-existing linear pathways on the territory [European Greenways Association 1999].

For these reasons, which relate to the different meanings that can be assumed and assigned, the greenways created and implemented these days often feature a structure, each differentiated from the others, depending on their characteristics and on the functions they serve [Mennella 2006].

Various differentiated greenways have been designed and created also in Italy.

The differences often derive from the use of greenways in territorial planning; indeed, the definition of greenway envisages a connection and relation among different values, entities, disciplines and subjects, thus covering and interlinking different themes ranging from those of a social, cultural and historical nature, to economic and territorial aspects.

Among the functions that may be served by greenways, there are also those connected with safeguarding the environment by structuring a series of intertwined lines with an ecological, functional and infrastructural connotation, involving large areas or regions.

As the strong "elements" of "open territories", greenways may cover small portions of protected areas as well as entire municipal plains of provincial and regional territories. They may even extend to $\mathrm{Eu}-$ ropean networks, thus creating evident physical and symbolic "marks" that enliven and characterise the territory as if fuelled by a desire to become independent entities, able to create... a functional network with the opportunity of connecting several entities (the natural and cultural resources of the landscape) of varying significance, at local, regional, national and European level, and pursuing multiple objectives by exploiting territorial characteristics and adjusting to the morphology of the places ... [Rizzo 2004]

This paper performs an analysis of interventions aimed at the creation of a greenway in Italy in order to ensure its consistency with the definitions of accredited organizations and associations. 

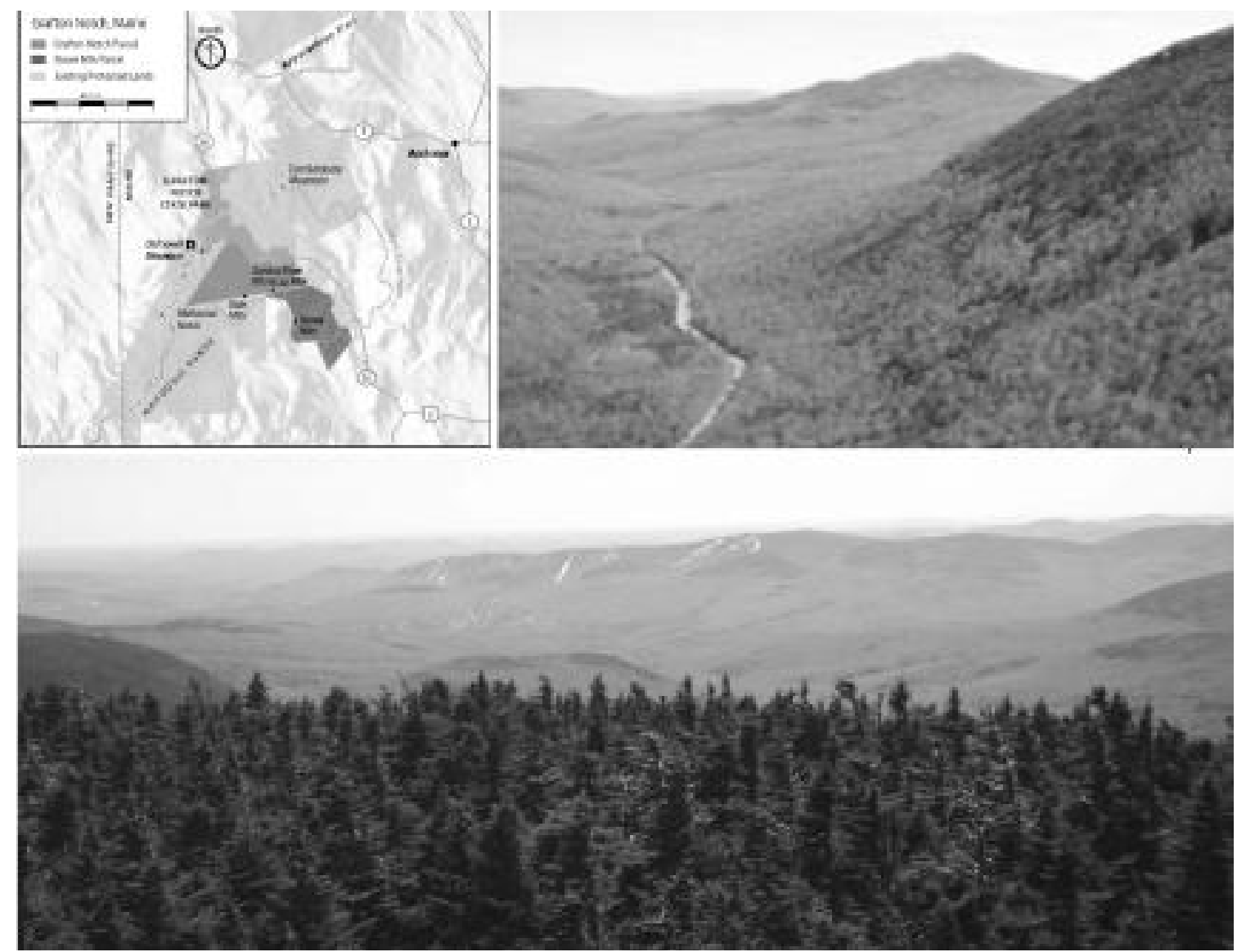

Fig. 1 - Example of a greenway in New England, Grafton Notch Landscape, (picture from A.T. Journeys, 32 July-August 2007).

\section{Materials and methods}

The specific dedicated bibliography was consulted to draw up this paper. Plans and projects were also examined, containing specific estimates of greenways, or in any case of alternative pathways (bike paths, green roads, etc.), and several sites were regularly inspected.

According to the method adopted, the official definitions of greenway were compared with the greenways and greenway "networks" examined across the country. For the purposes of comparison the characteristics and aims attributed to specific implementations and projects were summarised. The data referring to each examined intervention was summarised in a table in order to be able to make immediate comparisons and considerations.

The characteristics of the synthetic formulation of greenways on the table may become a reference by institutions and associations for cataloguing or for verification of contents and intervention procedures.

To analyse the state of the art, a summarising table was used, reporting the main characteristics of each intervention (defined as greenway or with similar wording, or those where the word greenway appears in the project or as part of the objectives pursued), with regards to the year of creation, the promoting body, the potential linear development (according to the project) as opposed to the actual development, the plans and the operating procedure for their creation, the procedures undertaken by the promoting bodies to access the funds, the type (urban, extra urban, naturalistic, or functional, recreational, educational, etc.) and finally the main features of the intervention, its objectives and the purposes pursued (table of interventions).

\section{Results and discussion}

The analysis and comparison of the data in the table of interventions reveal that the early greenways were set up between the end of the $20^{\text {th }}$ century and the early $21^{\text {st }}$ century, in line with an increasing interest in environmental issues and the concept of sustainability.

Of the 41 interventions, 24 have been implemented, while 9 are in their start-up phase and 8 constitute forecasts in the form of plans and specific projects.

An initial consideration that could be made, having examined the interventions, is that almost all of them derived from preliminary technical - scientific and social in-depth studies and, sometimes, from suitable feasibility studies, thus confirming the importance/ need of providing technical-scientific support for the proposal of interventions. 


\begin{tabular}{|c|c|c|c|c|}
\hline \multirow[t]{4}{*}{$\mathbf{N r}$} & Region & \multirow{4}{*}{$\begin{array}{l}\text { Inclusion in Plans and } \\
\text { projects }\end{array}$} & Type and dimensions & \multirow[t]{4}{*}{ References } \\
\hline & Name & & Objectives & \\
\hline & Year & & Creation & \\
\hline & Proposing bodv & & Characteristics & \\
\hline \multirow[t]{4}{*}{1} & Trentino Alto Adige & \multirow{4}{*}{$\begin{array}{l}\text { Proposed by "Associazione Italiana } \\
\text { Greenway" } \\
\text { and CO.MO.DO }\end{array}$} & Unused railway $50.5 \mathrm{Km}$ & \multirow[t]{4}{*}{ www.greenwaysitalia.it } \\
\hline & Greenway unused railway Ora-Predazzo & & Tourist valorisation & \\
\hline & 2003 & & Partial $25 \mathrm{Km}$; & \\
\hline & Company for tourism in the Val di Fiemme & & Urban - extra urban & \\
\hline \multirow[t]{4}{*}{2} & Piemonte & \multirow{4}{*}{$\begin{array}{l}\text { Plan for Cycling Itineraries of the } \\
\text { Municipality of Turin }\end{array}$} & Along the banks of the Po & \multirow[t]{4}{*}{ Municipality of Turin Map of cycle routes } \\
\hline & Greenway along the banks of Po river & & Functional valorisation for free time & \\
\hline & 2004 & & Total $16-18 \mathrm{Km}$ & \\
\hline & Municipality of Turin & & Urban-suburban-extra-urban & \\
\hline \multirow[t]{4}{*}{3} & Friuli Venezia Giulia - Veneto & \multirow{4}{*}{$\begin{array}{l}\text { Project- Gal Venezia Orientale with } \\
\text { Leader plus community funding }\end{array}$} & Old route, trunk road and towpath $15 \mathrm{Km}$ & \multirow[t]{4}{*}{ Pilot project "Gira Livenza" } \\
\hline & The greenway on the river Livenza and "Gira Livenza" & & Naturalistic routes, hydraulic works, wharfs and landings, cycle routes & \\
\hline & 2007 & & Total $15 \mathrm{~km}$ & \\
\hline & $\begin{array}{l}\text { Municipalities of Torre di Mosto and Caorle, Provinces of } \\
\text { Venezia, Treviso and Pordenone } \\
\end{array}$ & & Suburban-extra-urban & \\
\hline \multirow[t]{4}{*}{4} & Friuli Venezia Giulia & \multirow{4}{*}{$\begin{array}{l}\text { Projects financed by INTERREG III } \\
\text { Italy-Austria }\end{array}$} & Greenway and water ways $400 \mathrm{Km}$ & \multirow[t]{4}{*}{ Project INTERREG III Italia-Austria } \\
\hline & Pordenone Greenway & & The way of naturalistic, food and wine tourism & \\
\hline & 2000 & & Total $400 \mathrm{Km}$ & \\
\hline & Regional board and Coldiretti & & Extra-urban-suburban & \\
\hline \multirow[t]{4}{*}{5} & Friuli Venezia Giulia & \multirow{4}{*}{\begin{tabular}{|l|} 
Proposed by "Associazione Italiana \\
Greenway" and State public railways
\end{tabular}} & Unused railway & \multirow[t]{4}{*}{ www.greenwaysitalia.it } \\
\hline & Greenway unused railway of Val Rosandra (Trieste-Erpelle); & & Greenway, cycle routes & \\
\hline & 2010 & & about $17 \mathrm{Km}$ & \\
\hline & Municipality of Trieste & & Urban, suburban & \\
\hline \multirow[t]{4}{*}{6} & Veneto (Treviso) & \multirow{4}{*}{$\begin{array}{l}\text { Environmental Plan (2000); } \\
\text { "Varianti di Settore" (2004); } \\
\text { Projects Gal Venezia Orientale }\end{array}$} & $\begin{array}{l}\text { Unused railway (Treviso-Ostiglia } 10 \mathrm{~km} \text { with the recovery of the steam train) } \\
\text { and river towpath (Sile) } 100 \mathrm{Km}\end{array}$ & \multirow[t]{4}{*}{ http://www.tg0.it } \\
\hline & Greenway of Sile "Gira Sile" & & Equip and make accessible the naturalistic route in a park area, educational uses & \\
\hline & $2000-2004$ & & Itinerary of about $100 \mathrm{Km}$ & \\
\hline & Province of Treviso, Ente Parco Naturale Regionale del Sile & & Extra-urban, naturalistic route & \\
\hline \multirow[t]{4}{*}{7} & Veneto & \multirow{4}{*}{$\begin{array}{l}\text { Greenways association Italian; } \\
\text { Confederation Sustainable Mobility } \\
\text { (Co.Mo.Do);Federation parks; } \\
\text { Italian tourist railways }\end{array}$} & Unpaved route along the unused railway Rocchette-Asiago & \multirow{4}{*}{$\begin{array}{l}\text { Italian Greenways Association- } \\
\text { www.greenwaysitalia.it }\end{array}$} \\
\hline & Greenway unused railway Rocchette-Asiago & & Greenway & \\
\hline & 2009 & & About $27 \mathrm{Km}$ & \\
\hline & Associazione Artigiani di Asiago & & Urban, suburban, -extra-urban & \\
\hline \multirow[t]{4}{*}{8} & Veneto (Dolomiti) & \multirow{4}{*}{$\begin{array}{l}\text { Proposed by "Associazione Italiana } \\
\text { Greenway" and State public railways }\end{array}$} & Unused railway $65 \mathrm{Km}$ & \multirow{4}{*}{$\begin{array}{l}\text { Italian Greenways Association- } \\
\text { www.greenwaysitalia.it }\end{array}$} \\
\hline & Greenway of the former railway Calalzo di Cadore -Cortina- Dobbiaco; & & Tourist and naturalistic valorisation, cycle route & \\
\hline & 2003 & & about $65 \mathrm{Km}$ & \\
\hline & Proposed by “Associazione Italiana Greenways" & & Extra-urban & \\
\hline
\end{tabular}




\begin{tabular}{|c|c|c|c|c|}
\hline \multirow[t]{4}{*}{$\mathrm{Nr}$} & Region & \multirow{4}{*}{$\begin{array}{l}\text { Inclusion in Plans and } \\
\text { projects }\end{array}$} & Type and dimensions & \multirow[t]{4}{*}{ References } \\
\hline & Name & & Objectives & \\
\hline & Year & & Creation & \\
\hline & Proposing bodv & & Characteristics & \\
\hline \multirow[t]{4}{*}{9} & Italy-Switzerland & \multirow{4}{*}{$\begin{array}{l}\text { Programma Interreg IIIA, Progetto } \\
\text { Regionale Misura: } 2.3 \text {-Integration } \\
\text { and improvement of infrastructure } \\
\text { and transport systems }\end{array}$} & Unused railway $52 \mathrm{Km}$ & \multirow[t]{4}{*}{ http://www.interreg-italia svizzera.it; } \\
\hline & $\begin{array}{l}\text { Greenway of the railway Valmorea tract Mendrisio (Svizzera) e Malnate } \\
\text { (Italia) }\end{array}$ & & Tourist and naturalistic valorisation, steam train restoration; cycle route & \\
\hline & 2001-2002 & & $52 \mathrm{Km}$ & \\
\hline & $\begin{array}{l}\text { Work Group constituted by Councillor's Office of the } \\
\text { Lombardy Region Transportation, Province (Varese,Como), Helvetica } \\
\text { Club of S.Gottardo and Association railway Valmorea }\end{array}$ & & Extra-urban & \\
\hline \multirow[t]{4}{*}{10} & Liguria & \multirow{4}{*}{$\begin{array}{l}\text { Proposed by Region and Province } \\
\text { and "area } 24 \text { " private company }\end{array}$} & unused railway & \multirow[t]{4}{*}{ Società ligure Area 24} \\
\hline & Greenway between San Lorenzo e Santo Stefano & & Development (environmental tourism ) of unused areas, cycle routes & \\
\hline & 2008 & & $24 \mathrm{Km}$ & \\
\hline & Regione Liguria & & Urban-suburban & \\
\hline \multirow[t]{4}{*}{11} & Liguria & \multirow{4}{*}{$\begin{array}{l}\text { Project Poseidon (INTERREG III C) } \\
\text { Masterplan-Valle Scrivia Partner } \\
\text { europei (Amsterdam, la London } \\
\text { Haringey, North Kent) }\end{array}$} & Greenway & \multirow[t]{4}{*}{ Masterplan della valle Scrivia } \\
\hline & Greenway tra monti e mare Poseidon in valle Scrivia & & Neighbourhood management- urban and social recovery of disadvantaged areas & \\
\hline & $2004-2006$ & & & \\
\hline & $\begin{array}{l}\text { Medway-, Stockholm, Vienna and Province of Genoa Municipalities of } \\
\text { the Scrivia valley }\end{array}$ & & Urban-suburban-extra urban & \\
\hline \multirow[t]{4}{*}{12} & Liguria & \multirow{4}{*}{$\begin{array}{l}\text { Competition of ideas for the } \\
\text { recovery of the ex-area of the } \\
\text { steelworks- Master plan editing }\end{array}$} & Greenway & \multirow[t]{4}{*}{ Masterplan valley del Polcevora } \\
\hline & Greenway della valle del Polcevora & & Stitch together a degraded urban fabric, ecological networks at a local level & \\
\hline & 1999-2007 & & & \\
\hline & Municipality of Genoa; soc. Cornigliano & & Urban-suburban-extra-urban & \\
\hline \multirow[t]{4}{*}{13} & Liguria & \multirow{4}{*}{$\begin{array}{l}\text { Proposal of Municipalities for a } \\
\text { provincial network of cycle paths }\end{array}$} & Unused railway, coastal route, mountain route & \multirow[t]{4}{*}{ cycle network Savona } \\
\hline & $\begin{array}{l}\text { Greenway dell'arco ligure, Via dei Pellegrini,Via Pedemontana, Via del } \\
\text { Mare,Via dei Monti }\end{array}$ & & $\begin{array}{l}\text { Cycle route, cycle link between the province of Savona and the national and } \\
\text { European cycle net }\end{array}$ & \\
\hline & 2010 & & & \\
\hline & Province of Savona & & Urban-suburban-extra urban & \\
\hline \multirow[t]{4}{*}{14} & Lombardia & \multirow{4}{*}{$\begin{array}{l}\text { Proposed by "Associazione Italiana } \\
\text { Greenway" }\end{array}$} & Unused railway $40 \mathrm{Km}$ & \multirow[t]{4}{*}{ www.greenwaysitalia.it } \\
\hline & Greenway dell'ex ferrovia del Brembana & & Naturalistic valorisation of the route, cycle route & \\
\hline & 2007 & & Partial $24 \mathrm{Km}$ & \\
\hline & Municipality of Bergamo & & Urban-Extra urban & \\
\hline \multirow[t]{4}{*}{15} & Lombardia & \multirow[t]{4}{*}{ Regional Landscape Territorial Plan } & Martesana canal & \multirow{4}{*}{$\begin{array}{l}\text { Associazione Italiana Greenways; Ambiente e } \\
\text { Territorio June } 2007 \mathrm{nr} .3\end{array}$} \\
\hline & Greenway del Naviglio della Martesana & & Functional recovery for free time; cycle route & \\
\hline & 1999 & & $35 \mathrm{Km}$ & \\
\hline & Lombardy regional board & & Urban- suburban- extra-urban & \\
\hline
\end{tabular}




\begin{tabular}{|c|c|c|c|c|}
\hline \multirow[t]{4}{*}{$\mathbf{N r}$} & Region & \multirow{4}{*}{$\begin{array}{l}\text { Inclusion in Plans and } \\
\text { projects }\end{array}$} & Type and dimensions & \multirow[t]{4}{*}{ References } \\
\hline & Name & & Obiectives & \\
\hline & Year & & Creation & \\
\hline & Proposing bodv & & Characteristics & \\
\hline \multirow[t]{4}{*}{16} & Lombardia & \multirow[t]{4}{*}{ Regional Landscape Territorial Plan } & Great Canal $50 \mathrm{Km}$ & \multirow{4}{*}{$\begin{array}{l}\text { Associazione Italiana Greenway Ambiente } \\
\text { Territorio June } 2007 \mathrm{nr} .3\end{array}$} \\
\hline & Greenway del Naviglio Grande & & Functional recovery for free time; cycle route & \\
\hline & 1999 & & about $20 \mathrm{Km}$ & \\
\hline & Lombardy regional board - Municipality of Milan & & Urban- suburban- extra-urban & \\
\hline \multirow[t]{4}{*}{17} & Lombardia & \multirow{4}{*}{$\begin{array}{l}\text { Agenda Locale } 21 \text { - Pavia - } \\
\text { Programme for Sustainable } \\
\text { Development }\end{array}$} & Greenway $20 \mathrm{Km}$ & \multirow[t]{4}{*}{ http://agenda21.comune.pv.it } \\
\hline & Greenway della Battaglia & & $\begin{array}{l}\text { Functional itinerary for free time, environmental education and study of historic } \\
\text { buildings }\end{array}$ & \\
\hline & 2000 & & Partial 20 - Total $40 \mathrm{Km}$ & \\
\hline & $\begin{array}{l}\text { Municipality of Pavia Councillor's Office for the } \\
\text { Environment and Sustainable Development }\end{array}$ & & $\begin{array}{l}\text { Urbana-extra urban, Green path between Certosa and } \\
\text { the city of Pavia }\end{array}$ & \\
\hline \multirow[t]{4}{*}{18} & Lombardia & \multirow{4}{*}{$\begin{array}{l}\text { Sponsored by the Fondazione del } \\
\text { Monte Lombardia }\end{array}$} & Green route $120 \mathrm{Km}$ & \multirow[t]{4}{*}{ http://agenda21.comune.pv.it } \\
\hline & Greenway Milano-Pavia-Varzi & & $\begin{array}{l}\text { Sustainable Tourism educational-recreational; naturalistic and agricultural } \\
\text { resource development }\end{array}$ & \\
\hline & 2004 & & Itinerary of $120 \mathrm{Km}$ & \\
\hline & Lombardy regional board & & Urban-extra-urban-suburban & \\
\hline \multirow[t]{4}{*}{19} & Lombardia & \multirow{4}{*}{$\begin{array}{l}\text { The company Pedemontana } \\
\text { Lombarda, Politecnico di Milano } \\
\text { (DIAP) - Environmental Impact } \\
\text { Assessment }\end{array}$} & Greenway “area” $90 \mathrm{Km}$ & \multirow[t]{4}{*}{ http://www.alternativaverde.it; } \\
\hline & Greenway della Pedemontana & & Compensation works for highway project & \\
\hline & 2008 & & Itinerary of $90 \mathrm{Km}$ & \\
\hline & Provinces of Bergamo and Varese, Soc. Pedemontana spa & & Urban-suburban-extra-urban & \\
\hline \multirow[t]{4}{*}{20} & Lombardia & \multirow{4}{*}{$\begin{array}{l}\text { Proposed by the Provinces of } \\
\text { Brescia-Milano and Lecco }\end{array}$} & Greenway $320 \mathrm{Km}$ & \multirow[t]{4}{*}{ Protocol of understanding among the provinces } \\
\hline & Greenway percorsi tra Brescia-Milano-Lecco & & Cycle routes Extension of the Martesana greenway & \\
\hline & 2008 & & In project & \\
\hline & Provinces of Brescia; Milano; Lecco & & Urban-suburban-extra-urban & \\
\hline \multirow[t]{4}{*}{21} & Lombardia & \multirow[t]{4}{*}{ Plan cycle network of valleys } & Unused railway $32 \mathrm{Km}$ & \multirow{4}{*}{$\begin{array}{l}\text { Associazione Italiana Greenway } \\
\text { www.greenwaysitalia.it }\end{array}$} \\
\hline & Greenway della ex ferrovia Val Seriana & & Functional valorisation for free time; cycle route & \\
\hline & 2003 & & Partial $13 \mathrm{Km}$ & \\
\hline & Municipality of Bergamo & & Urban - extra-urban & \\
\hline \multirow[t]{4}{*}{22} & Lombardia & \multirow{4}{*}{$\begin{array}{l}\text { Single document of Objective } \\
\text { Planning } 2 \text { (Strengthening and } \\
\text { improvement of infrastructural } \\
\text { equipment for sustainable } \\
\text { development) }\end{array}$} & Greenway $10 \mathrm{Km}$ & \multirow[t]{4}{*}{ Associazione italiana Greenways } \\
\hline & Greenway del lago di Como & & Tourist revaluing of the lake of Como & \\
\hline & 2002-2007 & & Total $10 \mathrm{~km}$ & \\
\hline & Fondazione Banca Lombardia & & Urban - suburban & \\
\hline \multirow[t]{4}{*}{23} & Lombardia & \multirow{4}{*}{$\begin{array}{l}\text { Local Development Integrated Plan } \\
\text { (PISL) }\end{array}$} & Cycle routes & \multirow[t]{4}{*}{ http://www.valleolona.org } \\
\hline & Greenway Medio Olona & & Associate environmental protection with industrial economic development. & \\
\hline & 2003-2004 & & & \\
\hline & Municipalities of Fagnano Olona at all. & & Urban-suburban-extra urban & \\
\hline
\end{tabular}




\begin{tabular}{|c|c|c|c|c|}
\hline \multirow[t]{4}{*}{$\mathbf{N r}$} & Region & \multirow{4}{*}{$\begin{array}{l}\text { Inclusion in Plans and } \\
\text { projects }\end{array}$} & Type and dimensions & \multirow[t]{4}{*}{ References } \\
\hline & Name & & Objectives & \\
\hline & Year & & Creation & \\
\hline & Proposing bodv & & Characteristics & \\
\hline \multirow[t]{4}{*}{24} & Lombardia & \multirow{4}{*}{$\begin{array}{l}\text { VAS del PTCP-Provincia di Lodi - } \\
\text { Piano di indirizzo forestale- } \\
\text { Rapporto Ambientale, Progetto } \\
\text { Rever Med (Rete verde europea, } \\
\text { finanziato dall'Unione Europea). } \\
\end{array}$} & Greenway along the canal Muzza & \multirow[t]{4}{*}{ www.greenwaysitalia.it } \\
\hline & greenway del canale della Muzza & & Greenway, cycle route & \\
\hline & 2009 & & $72 \mathrm{Km}$ & \\
\hline & Province of Lodi & & Suburban, -extra-urban & \\
\hline \multirow[t]{4}{*}{25} & Lombardia & \multirow{4}{*}{$\begin{array}{l}\text { Progetto Integrato d'Area between } \\
\text { Parco Adda Nord and Province of } \\
\text { Milan }\end{array}$} & Route along the canal Adda & \multirow{4}{*}{$\begin{array}{l}\text { www.greenwaysitalia.it } \\
\text { www.parcoaddanord.it }\end{array}$} \\
\hline & Greenway del Parco Adda Nord & & Greenway, cycle route & \\
\hline & 2003 & & $30 \mathrm{Km}$ & \\
\hline & Region Lombardy and Adda Nord River & & Suburban,-extra-urban & \\
\hline \multirow[t]{4}{*}{26} & Emilia Romagna & \multirow{4}{*}{$\begin{array}{l}\text { Province Modena.Programma di } \\
\text { valorizzazione e promozione del } \\
\text { territorio (P.V.P.T.) }\end{array}$} & Unused railway $21 \mathrm{Km}$ & \multirow[t]{4}{*}{ www.greenwaysitalia.it } \\
\hline & Greenway dell'ex ferrovia Modena-Vignola & & path for the development and study of historic buildings cycle route & \\
\hline & 2004 & & Total $21 \mathrm{Km}$ & \\
\hline & Municipality of Modena & & Urban- suburban- extra-urban & \\
\hline \multirow[t]{4}{*}{27} & Emilia Romagna & \multirow{4}{*}{$\begin{array}{l}\text { Piano di Coordinamento Provinciale } \\
\text {-"Rete per la Sicurezza" Provincia di } \\
\text { Modena }\end{array}$} & Unused railway & \multirow{4}{*}{$\begin{array}{l}\text { Associazione Italiana Greenway; Provincia di } \\
\text { Modena }\end{array}$} \\
\hline & Pista ciclabile-Modena-Villafranca-Mirandola & & $\begin{array}{l}\text { Cycle route, reuse of the buildings of the railway station, connection between } \\
\text { urban centers and neighboroughs, safe mobility }\end{array}$ & \\
\hline & 2005 & & $40 \mathrm{Km}$ & \\
\hline & Regione Emilia Romagna, Provincia di Modena & & Urban-extra-urban & \\
\hline \multirow[t]{4}{*}{28} & Toscana & \multirow{4}{*}{$\begin{array}{l}\text { Project to reduce the hydraulic risk } \\
\text { of Vingone stream and create a } \\
\text { green path for leisure }\end{array}$} & Alzaia del Vingone $13 \mathrm{Km}$ & \multirow{4}{*}{$\begin{array}{l}\text { Press Office of the Consorzio Bonifica delle } \\
\text { colline del Chianti }\end{array}$} \\
\hline & Greenway del Vingone & & $\begin{array}{l}\text { Project to decrease the hydraulic risk of the river Vingone and to become a } \\
\text { urban riverside greenway -recreational for slow mobility }\end{array}$ & \\
\hline & 2006 & & $13 \mathrm{Km}$ projected & \\
\hline & Province of Florence and the Municipalities of Scandicci & & Urban-suburban-extra-urban & \\
\hline \multirow[t]{4}{*}{29} & Umbria & \multirow{4}{*}{$\begin{array}{l}1990 \text { Soc. Spolentina - Restoration } \\
\text { and valorisation project of the } \\
\text { railway line for tourist purposes. A } \\
\text { project to restore the buildings in the } \\
\text { Municipalities crossed by the } \\
\text { railway }\end{array}$} & Unused railway; $51 \mathrm{Km}$ & \multirow{4}{*}{$\begin{array}{l}\text { XII intern. meeting "Desire, freedom and need } \\
\text { in creating a cultural landscape mosaic" - } \\
\text { Cividale del Friuli (Ud), } 25,26 \text { October } 2007 \text { - } \\
\text { University of Perugia }\end{array}$} \\
\hline & Tracciato della ferrovia dismessa Norcia-Spoleto & & Valorise the former railway track & \\
\hline & 2000 & & Partial - circa $12.5 \mathrm{Km}$ & \\
\hline & Società Spolentina & & Urban-extra urban & \\
\hline \multirow[t]{4}{*}{30} & Lazio & \multirow[t]{4}{*}{ Lazio Regional Law n. 40/99 } & Unused railway Roma-Fiuggi-Paliano, $(22 \mathrm{Km})$ & \multirow[t]{4}{*}{ www.greenwaysitalia.it } \\
\hline & Pista ciclabile dell'ex ferrovia Fiuggi-Paliano & & Cycle route & \\
\hline & 2003 & & $22 \mathrm{Km}$ & \\
\hline & Regione Lazio & & Extra-urban/naturalistic & \\
\hline \multirow[t]{4}{*}{31} & Abruzzo & \multirow[t]{4}{*}{ Initiative of the Regione Abruzzo } & Unused railway $46 \mathrm{Km}$ & \multirow{4}{*}{$\begin{array}{l}\text { Proposed laws by the Region for the formation } \\
\text { of a natural reserve along the coast of Teatina. } \\
\text { Protocol } \\
\text { of understanding for the acquisition of the areas }\end{array}$} \\
\hline & Greenway Vasto-San Salvo & & Revaluing the coast strip, cycle routes & \\
\hline & 2005 & & In project $46 \mathrm{Km}$ & \\
\hline & Abruzzo regional and provincial boards, Municipalities & & Suburban- Extra-urban & \\
\hline
\end{tabular}




\begin{tabular}{|c|c|c|c|c|}
\hline \multirow[t]{4}{*}{$\mathbf{N r}$} & Region & \multirow{4}{*}{$\begin{array}{l}\text { Inclusion in Plans and } \\
\text { projects }\end{array}$} & Type and dimensions & \multirow[t]{4}{*}{ References } \\
\hline & Name & & Objectives & \\
\hline & Year & & Creation & \\
\hline & Proposing bodv & & Characteristics & \\
\hline \multirow[t]{4}{*}{32} & Campania & \multirow{4}{*}{$\begin{array}{l}\text { Project Feasibility Executive Project } \\
\text { created between the public and } \\
\text { private sectors } \\
\text { (Province of Salerno, the } \\
\text { Municipalities of } \\
\text { Torchiara:Fondazione Megalia }\end{array}$} & Greenway $15 \mathrm{Km}$ & \multirow{4}{*}{$\begin{array}{l}\text { Text taken from "The Green Way Torchiara - } \\
\text { Greenway: a gentle path through history and } \\
\text { nature". Fondazione Megalia }\end{array}$} \\
\hline & La verda via di Torchiara & & $\begin{array}{l}\text { Protect the historical and natural heritage, tourist revaluation to increase the } \\
\text { economic and social growth }\end{array}$ & \\
\hline & 2004 & & $15 \mathrm{Km}$ & \\
\hline & Province of Salerno, the Municipalities of Torchiara; Fondazione Megalia & & Suburban- extra-urban & \\
\hline \multirow[t]{4}{*}{33} & Campania-Basilicata & \multirow{4}{*}{$\begin{array}{l}\text { Initiative proposed by Università } \\
\text { Popolare della Terza Età (Polla) and } \\
\text { Club Alpino Italiano (Salerno); }\end{array}$} & Unused railway $76 \mathrm{Km}$ & \multirow[t]{4}{*}{ www.ferroviedimenticate.it } \\
\hline & Greenway Lagonegro-Sicignano & & To avoid the depopulation and cultural and social loss of villages situated inland & \\
\hline & 2008 & & In project $76 \mathrm{Km}$ & \\
\hline & $\begin{array}{l}\text { Associazione ferrovia dimenticata- Università popolare della Terza Età- } \\
\text { Club Alpino Italiano }\end{array}$ & & Suburban-extra-urban & \\
\hline \multirow[t]{4}{*}{34} & Basilicata & \multirow{4}{*}{$\begin{array}{l}\text { Guidelines for the promotion of the } \\
\text { tourist areas crossed by a number of } \\
\text { former railway lines in Basilicata, } \\
\text { Calabria, Sardinia }\end{array}$} & Unused railway $42 \mathrm{Km}$ & \multirow{4}{*}{$\begin{array}{l}\text { Iniziativa Comunitaria Equal II fase } \\
\text { Progetto "Le Vie del Treno" } \\
\text { www.leviedeltreno.it }\end{array}$} \\
\hline & Greenway Potenza-Laurenzana & & $\begin{array}{l}\text { Avoid the depopulation and cultural and social loss of villages situated inland, } \\
\text { through tourist promotional initiatives; cycle routes }\end{array}$ & \\
\hline & 2007 & & In project $42 \mathrm{Km}$ & \\
\hline & $\begin{array}{l}\text { European Social fund of the European Union, Ministry of employment } \\
\text { and social welfare, Equal community initiative }\end{array}$ & & Urban, suburban, -extra-urban & \\
\hline \multirow[t]{4}{*}{35} & Puglia & \multirow{4}{*}{$\begin{array}{l}\text { Integrated Programme for urban and } \\
\text { environmental redevelopment } \\
\text { of peripheral areas }\end{array}$} & Routes/cycle route & \multirow[t]{4}{*}{ www.Regione Puglia.it } \\
\hline & Greenway Manfredonia & & Urban and environmental redevelopment of peripheral areas & \\
\hline & 2008 & & In project & \\
\hline & Municipality of Manfredonia-Foggia Province & & Urban-suburban & \\
\hline \multirow[t]{4}{*}{36} & Puglia & \multirow{4}{*}{$\begin{array}{l}\text { Protocol of understanding between } \\
\text { Regional Councillors office for } \\
\text { Transport of the Regione Puglia and } \\
\text { Acquedotto pugliese-Progetto } \\
\text { CYRONMED (Cycle Route } \\
\text { Network of the Mediterranean } \\
\text { financed with Interreg III B } \\
\text { Archimed funds }\end{array}$} & Service lines of the Apulian aqueduct $244 \mathrm{Km}$ subdivided in 5 itineraries & \multirow[t]{4}{*}{ www.cicloamici.it/vie_verdi_in_puglia.htm } \\
\hline & Le vie "verdi" di Puglia & & $\begin{array}{l}\text { Cycle routes to valorisation of historical centres of cultural interest and typical } \\
\text { products }\end{array}$ & \\
\hline & 2008 & & In project & \\
\hline & $\begin{array}{l}\text { Puglia regional board - Regional Councillors office for Transport - } \\
\text { Sustainable Mobility Service and cycling (FIAB) }\end{array}$ & & suburban, extra-urban & \\
\hline \multirow[t]{4}{*}{37} & Sicilia & \multirow{4}{*}{\begin{tabular}{|l|} 
Regional Plan for Transports; \\
Feasibility study proposed by \\
Associazione italiana Greenways
\end{tabular}} & $\begin{array}{l}\text { Unused mining railway, Circuit of the Erei Nebrodi, Dittaino - Leonforte - } \\
\text { Nicosia; Circuit of the mines; Circuit of the lake; }(42 \mathrm{Km})\end{array}$ & \multirow{4}{*}{$\begin{array}{l}\text { Conference of } 20 \text { October } 2003 \text { "Valorising } \\
\text { unused railway lines through the creation of a } \\
\text { system of Greenways", Italian State Railway } \\
\text { ISFORT }\end{array}$} \\
\hline & Greenway degli Erei & & Tourist valorisation, an incentive to local micro economies, cycle routes & \\
\hline & 2003 & & Partial-9 Km & \\
\hline & Sicily regional board - Province of Enna - Association Greenway & & Extra urban - agricultural naturalist routes through the hills & \\
\hline \multirow[t]{4}{*}{38} & Sicilia & \multirow{4}{*}{$\begin{array}{l}\text { Redevelopment and revaluation of } \\
\text { internal protected areas, an incentive } \\
\text { to local micro economies, cycle } \\
\text { routes }\end{array}$} & About $42 \mathrm{Km}$ & \multirow{4}{*}{$\begin{array}{l}\text { Conference of } 20 \text { October } 2003 \text { "Valorising } \\
\text { unused railway lines through the creation of a } \\
\text { system of Greenways", Italian State Railway - } \\
\text { ISFORT }\end{array}$} \\
\hline & Greenway of the unused railway Siracusa-Vizzini & & $\begin{array}{l}\text { Integrated Plan of Sustainable Development of the Valleys of Anapo del } \\
\text { Cassile, Gal Valle dell'Anapo (Local Action Group); }\end{array}$ & \\
\hline & 2003 & & & \\
\hline & Sicily regional board & & Perceptive naturalistic route & \\
\hline
\end{tabular}




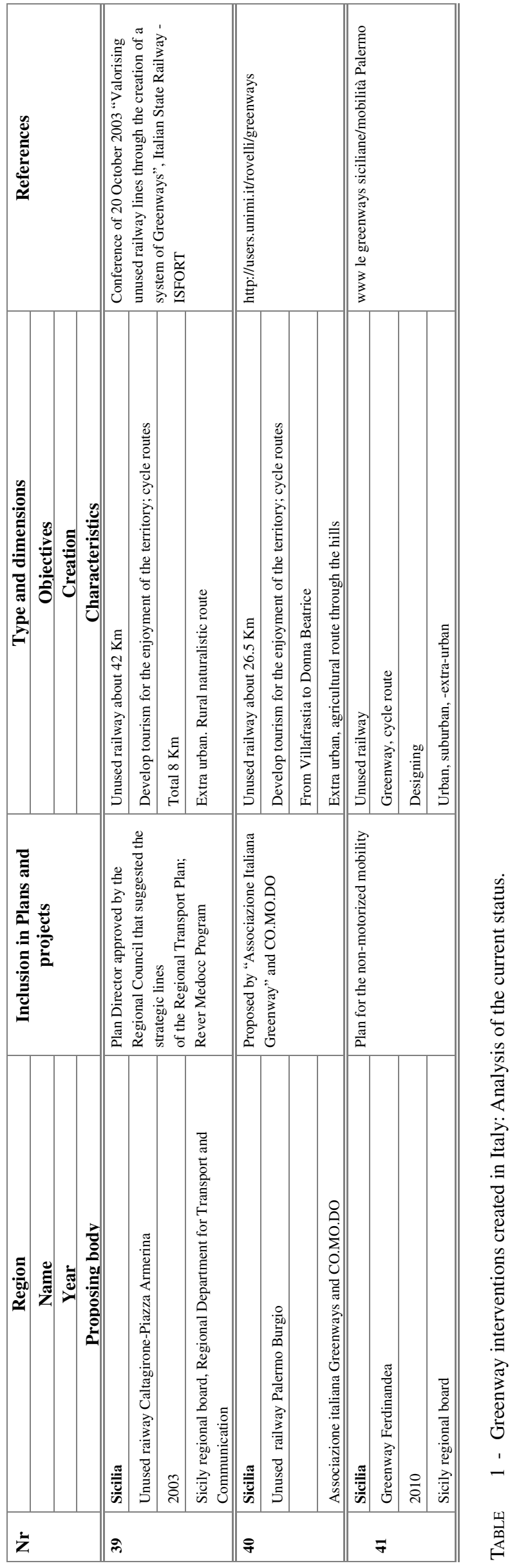

The region boasting the highest number of implemented interventions is the Lombardy region with 12 greenways, followed by Sicily with 5 interventions, mainly connected to the Rever Med project, a European project with the aim of creating a huge Green Network along the tracks of unused railways, for the entire western Mediterranean area, connecting and involving Portugal, Spain, France and Italy.

Liguria, with 4 greenway proposals of inter-municipal and European worth, constitutes a novelty in the group of the sector initiatives, since it involves unused industrial and port areas.

There are eight interventions that are currently only proposals, 2 for Puglia, 2 for Sicily and one each for Abruzzo, Campania, Basilicata and Tuscany, while there are 23 implemented interventions that involve 12 regions. Worth noting for the originality is the proposal by the Puglia region to use the infrastructural network of the Apulian aqueduct as a greenway.

Concerning relations with territoriality, it emerges that the implementations that are most in line with the official definitions of greenway -..." Greenways are systems or networks of connected lands that are protected, managed or developed to provide ecological, recreational and historical/cultural benefits" [Associazione Italiana Greenway], "From the mobility point of view, greenways are a system of routes, dedicated to a non-motorised traffic, connecting people with landscape resources (natural, historical, cultural, etc.) and the "centres of life", both in the urban areas and in the countryside" ... [Associazione Italiana Greenway] - are those of inter-provincial worth, where objectives also of a social - economic nature are pursued, mainly connected with the need to fight the phenomenon of disappearing small inland villages and control the unbalanced urban expansion in a metropolitan area.

Almost all of the proposals and interventions examined may be considered to be in line with the various definitions mentioned above, since they include, in general, many functions. For example, the greenways of the canals in Milan and those along the banks of the river Po in Turin, focus on the leisure function, though without neglecting the other aspects linked to education-knowledge, for the valorisation of the suburban and extra-urban territory.

Notable examples are the two greenways of Battaglia, in Pavia, and Milano-Pavia-Varzi. The latter, seen as an extension of the former, significantly recovers the historical traffic routes heading for the sea and restores river areas that today are still mostly occupied and cultivated without permission.

The Battaglia greenway in Pavia certainly is the most consistent with the historic and official definitions on the meaning of the term greenway. This consistency is shown, with respect to the interventions examined, by the fact that the pathway used was proposed as a route of historic-cultural and landscape worth, connecting together the most qualifying elements, for an organic use of the territory it belongs to. 
A route that introduces, and guides us through, the "discovery" of the landscape, the historical and monumental places and the cultural wealth of the territory of Pavia [Rizzo 2004; Valentini 2005].

In terms of pre-existing linear elements to be used as greenways, we found that unused railway lines very often provide a greater and immediate feasibility and usability, though conditioned with respect to the resources present along the route, which, however, generally have quite an important landscape value, as is the case for Spoleto-Norcia, Dobbiaco-Cortina, the Val Brembana, the former railway Ora-Predazzo, the former railway Modena-Vignola, the greenway of Val Seriana, the track of the former railway Mendrisio in Switzerland and Malnate in Italy.

The creations that are the least consistent with the definition of greenway concern those cases where the primary objective is exclusively connected with slow mobility (cycle lanes), since they are excessively single minded.

In any case, having ascertained that the main purposes to be pursued by a greenway are connected with slow or alternative mobility, and with the valorisation of the endogenous resources, without neglecting the ecological aspects, where present, all of the Italian initiatives examined, defined as greenways, may be considered as such, according to the most accredited definition in Europe and Italy.

Not a minor issue, which emerged from the analysis of the Italian initiatives, is related to the difficulties, by associations and organizations, to combine their skills and specificity to achieve common inter- ests. In fact, often collaboration is difficult between organizations with different motivations, breaking large-scale initiatives, sometimes even for simple matters of administrative boundaries.

This has affected the creations, breaking them despite their validity and functionality.

Furthermore, from examining the individual cases, it is clear how sometimes, in order to assign specific functions to greenways, designed for multiple functions, the will of individual cycling or environmentalist associations prevails over others, thus limiting the potential functions of the route.

For the interventions implemented in Sicily, instead, where decommissioned rail tracks serving old mines have been used, a right level of consistency was reached between the objectives to be followed and the way to create them, through the strong shared will of bodies and associations and the coordination among Municipalities, Provinces and Regions in setting the methods and procedures to implement the intentions.

In addition, it must be pointed out that the creation of greenways is not always easy due to problems connected with making the route safe for users, the availability of the entire railway line or of the linear element referred to, or due to the non accessibility to the surrounding areas.

With reference to the financed interventions [Agenda 21 Locale; Progetti Interreg; Gal; etc.], it emerged that those like the greenways of Pavia, Sicily and Pordenone, are among the most consistent with the accredited definitions of a greenway.

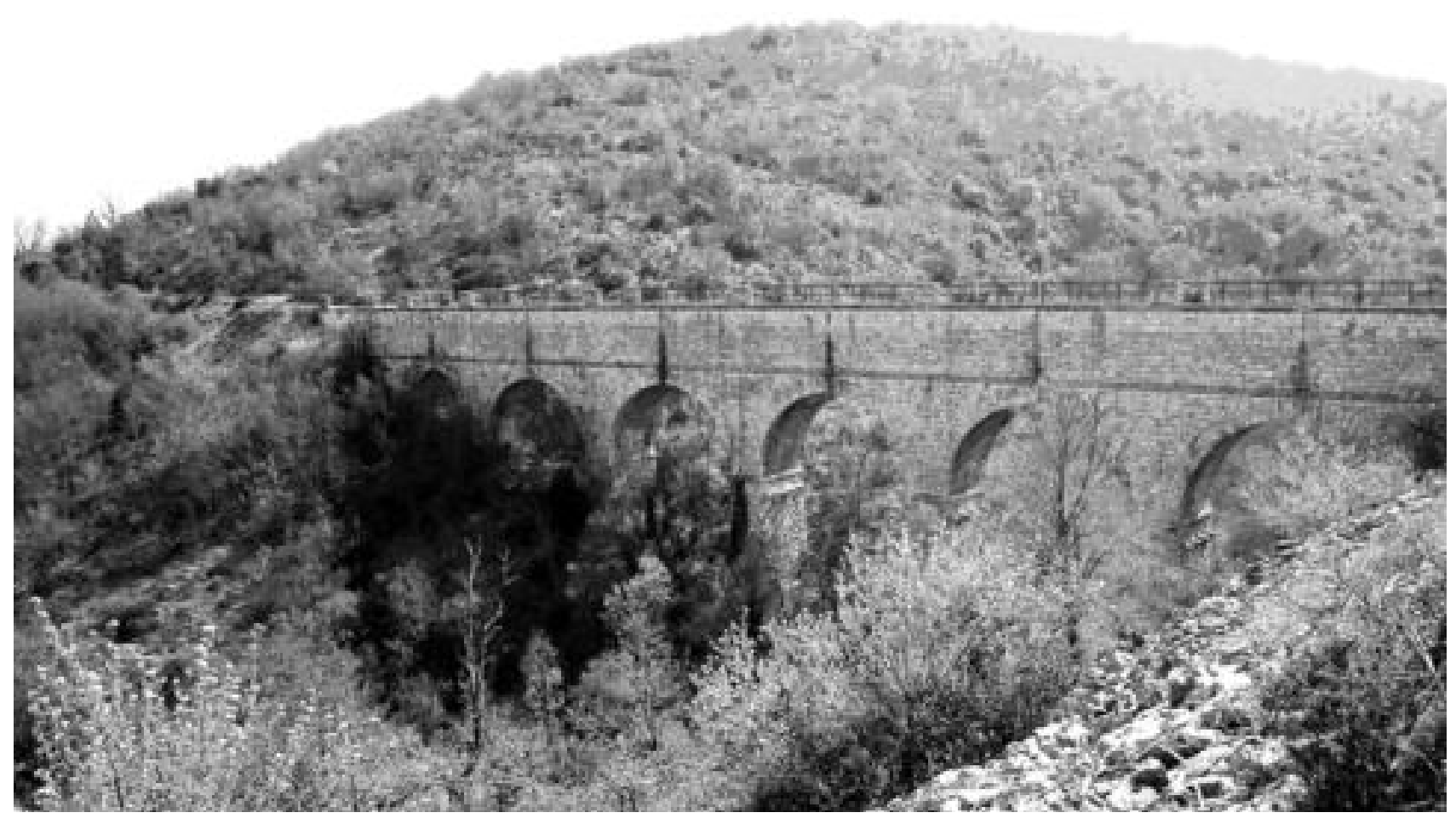

Fig. 2 - Greenway through a bridge and a canal along the Acquedotto Pugliese. (picture from "vie verdi" greenways in Puglia). 
Some creations (the cases of Genoa proposed in the master plans) attribute the creation of greenways to the possibility of finding a solution to issues concerning the urban and social recovery of disadvantaged areas or marginal and dismissed areas (former industrial zones), while respecting neighbouring areas of environmental worth (neighbourhood management) and, in general, of the maps AUDIS (Associazione Aree Urbane Dismesse).

It is also important to underline that, in some examples of greenways (the Pedemontana in Lombardy, the green path of Torchiara in Campania and the route of food and drink tourism in Friuli Venezia Giulia), the promoters and financers are private companies or associations, such as Coldiretti for Pordenone. This constitutes a tangible sign of how these interventions can increase credibility in the business world, with the possibility of profits being made - particularly for the agricultural sector -, just like the patronage that characterised the Renaissance. These measures tell us about the possibilities inherent in the definition of greenways especially if used for planning resources on the rural area affecting not only the historical and environmental-naturalistic but also productive and economic development.

\section{Conclusion}

The material used has made it possible for us to compare the characteristics of individual greenways with the official greenway definitions created in recent years, in a way to ascertain the project's adherence to and/or the moving away from the official definitions of a greenway. This operation has also given us an opportunity to reflect on the way in which greenways can be a tool supporting the planning and organization of rural areas, a system that can connect multiple issues relating to social and cultural aspects of transformations that affect rural landscape.

The most evident, consistent and ecological opportunities a greenway or a greenway network may offer as a tool to plan and transform the territory, are linked to the valorisation of the existing trails as well as to the heritage of rural architecture and food products (farm houses, rural houses, villages, roadman's houses, green tourism facilities, protection of typical and endangered productions) that is able to produce economies of scale at a local and regional level, and so on.

The greenway projects and creations, by promoting the use of eco-compatible means and methods, contribute to safeguarding the agricultural landscape, valorising agro-environmental and naturalistic resources, developing recreational and tourist forms of use, while providing an opportunity to sustain agricultural activities by favouring their environmental compatibility [Valentini 2005].

Indeed, the relationship between greenways and the rural territory is a key element for the actual feasi- bility of the projects. Greenways find in the agricultural landscape an ideal substrate for their development, where routes to discover, resources to be valorised and the living places to be connected, are all closely intertwined. They can become tools for a new organisation of the rural territory since, by integrating the objective of valorising the resources with the one of making them usable, they are able to channel the territorial policies towards a sustainable development, thus improving the connection between urban and extra urban environments and triggering mechanisms for the valorisation of the rural territory (green tourism, local trade, etc.).

In Italy a huge heritage of infrastructures that can be used for a new mobility is present. These can play a supporting role to the existing road network, dedicated only to motor traffic, which are environmentally friendly and, at the same time, able to support activities that affect rural areas.

\section{Acknowledgements}

The present work has been carried out under the Project Research of the University of Bari "La sostenibilità ambientale delle infrastrutture nell'assetto del territorio rurale".

\section{References}

Ahern J., Greenway as a planning strategy. Landscape and Urban Planning, 1995, 33, pp. 131-135.

Associazione Italiana Greenway: www.greenway.it.

Capobianco R.L., Tortora A., Picuno P., Analisi del territorio, dell'ambiente e del paesaggio rurale mediante tecniche di modellizzazione spaziale ed image processing di cartografie storiche. Rivista di Ingegneria Agraria, 2004, 35, (3), 71-77.

Dal Sasso P, Marinelli G., Ottolino M.A., Una rete di percorsi verdi per la valorizzazione e lo sviluppo del territorio rurale del Gargano Nord, In "Greenway per lo sviluppo sostenibile del territorio" a cura di Mennella V.G.G. Il Verde Editoriale, 2004, Milano.

European Greenways Association, The European Greenways Good Practice Guide, 1999.

Fabos J.G., Ahern J. (editors), Greenways. The beginning of an international movement. Elsevier,1995, Amsterdam.

Fabos J.G., Greenway planning in the United State: its origins and recent case studies. Landascape and Urban Planning, 2004, 68, pp. 321-342.

Fabos J.G., Ryan R.L., International greenway planning: an introduction. Landscape and Urban Planning, 2004

Guide Alleanza De Agostini Outdoor, Greenways in Italia. Scoprire le nostre strade verdi. De Agostini, 2003, Milano.

Yokohari M., Amemiya M., Amati M., The history and future directions of greenway in Japanese New Towns. Landscape and Urban Planning, 2006, 76, pp. 210-222.

Little C.E., Greenways for America. Johns Hopkins University Press, 1990, Baltimore (MD).

Maternini G., Pezzagno M., Le caratteristiche funzionali 
delle greenways: un primo approccio metodologico. $2^{\circ}$ Convegno Europeo Greenways, 1999, Milano 22 ottobre.

Mennella V.G.G. e altri, Profilo Italia Indicatori e modelli per lo sviluppo sostenibile del territorio e la valorizzazione del paesaggio. Ali\&no editrice, 2006, Perugia.

Rizzo G.G., Valentini A., Luoghi e paesaggi in Italia. Collana del Dottorato di ricerca in progettazione paesistica dell'Università degli Studi di Firenze, 2004, University Press Firenze.

Taylor J., Paine C., FitzGibbon J., From greembelt to greenways: four Canadian case studies. Landascape and Urban Planning, 1995, 33, pp. 47-64.

Toccolini A. e altri, Percorsi verdi: un'opportunità di sviluppo e riscoperta del territorio rurale. Quaderni I Georgofili, 2001, 1/2000, Firenze.

Toccolini A., Fumagalli N., Senes G., Valorizzazione delle linee ferroviarie non utilizzate attraverso la creazione di un sistema di greenways. Associazione greenway Italia, 2000, Milano.

Tortora A., Picuno P., Analysis of the effect on rural landscape of wide coverings for crop growing. Acta Horticulturae, 2008, vol. 801, pp. 325-332 ISSN: 0567-7572.

Turner T., Greenways: theory and history. Conferenza Facoltà di Agraria Università di Milano, 2001, 17 maggio.

Valentini A., Mettere in rete le risorse: le greenway quali strumenti per il progetto del paesaggio perturbano. In Quaderni della Ri-Vista Ricerche per la progettazione del paesaggio, Università degli Studi di Firenze, 2005, anno 2, numero 2, volume 2, maggio-agosto.

\section{SUMMARY}

The study analyses the greenway projects implemented in Italy, summarising their characteristics in a table that contains basic information concerning the geographical location, year of implementation, the type of layout and size, the state of implementation; the Institution that proposed the implementation, the official name, the territorial feature, the socio-economic and cultural aims, references to bibliographic and web resources and to their inclusion in plans and projects. The analysis has allowed to verify the compliance of individual contributions to the definitions attributed to the greenway from national and international associations. It has been possible to verify the use of greenways as physical support to spatial planning and the promotion of economic and productive development of rural areas.

Keywords: planning wide area - rural territory table of greenway. 
